\title{
Iron accumulation in human spleen in autoimmune thrombocytopenia and hereditary spherocytosis
}

\author{
Biro $\mathrm{C}^{1}$, Kopani $\mathrm{M}^{2,3}$, Kopaniova $\mathrm{A}^{4}$, Zitnanova $\mathrm{I}^{5}$, El-Hassoun $\mathrm{O}^{2}$, Minoo $\mathrm{P}^{6}$, Kolenova $\mathrm{L}^{2}$, \\ Sisovsky $\mathrm{V}^{2}$, Caplovicova $\mathrm{M}^{7}$, Stvrtina $\mathrm{S}^{2}$, Galfiova $\mathrm{P}^{8}$, Guller $\mathrm{L}^{9}$, Jakubovsky $\mathrm{J}^{2}$
}

Histopatologia Ltd, Bratislava, Slovakia. martin.kopani@fmed.uniba.sk

\begin{abstract}
Background: Although the iron is an essential element for the physiological functions of cells, tissues and organs, it is also an important inductor of reactive oxygen species (ROS).

Material and methods: Three groups of human spleen with autoimmune thrombocytopenia (AITP), hereditary spherocytosis (HS) and reference samples stained by haematoxylin and eosin, Perls' reaction for nonheme $\mathrm{Fe}(\mathrm{III})$ iron and Alcian blue for glycoconjugates detection were studied.

Results: Positive Perls' reaction in both AITP and HS groups was seen. Higher positivity in the HS than in AITP group was observed. HS group showed a higher amount of acidic glycoconjugates deposits than AITP group. Iron overload in HS and AITP leads to overproduction of ROS.

Conclusion: We suggest that acidic glycoconjugates deposits are involved in antioxidant defence by elimination and restriction of iron as a ROS inducer (Fig. 4, Ref. 19). Full Text in PDF www.elis.sk.

Key words: spleen, autoimmune thrombocytopenia, hereditary spherocytosis, iron, reactive oxygen species.
\end{abstract}

Iron occurs in the lithosphere and the pedosphere, as well as in the biosphere. It can influence iron metabolism, magnetotaxis, biological structure of cells and tissues to a certain extent. Existing knowledge concerning the presence of iron in the human body comes mainly from the results of measurements by nuclear magnetic resonance (NMR). The disadvantage of gained results is that it offers only qualitative information about iron in examined tissue. Histochemical methods have higher sensitivity and higher rate of specificity than NMR. This can be essential for understanding the role of iron in organism. Alcian blue is one of the most widely used cationic dye. Acidic glycoconjugates detected by Alcian blue are brown-black (1). Alcian blue bounds to negative surface of red blood cells (RBC) corresponding to sialic acid content. As a result of structural changes in various pathological diseases its affinity to the surface of RBC is changed (2), resulting in brown color of RBC.

In the present study, we histochemically studied distribution of nonheme ferric Fe(III) iron deposits in human spleen with he-

${ }^{1}$ Histopatologia Ltd, Bratislava, Slovakia, ${ }^{2}$ Comenius University, Faculty of Medicine, Institute of Pathology, Bratislava, Slovakia, ${ }^{3}$ Institute of Laboratory Medicine, St. Elizabeth University of Health and Social Sciences, Bratislava, Slovakia, ${ }^{4} 2$ nd Department of Neurology, Derer's Hospital, Bratislava, Slovakia, ${ }^{5}$ Comenius University, Faculty of Medicine, Institute of Medical Chemistry, Biochemistry and Clinical Biochemistry, Bratislava, Slovakia, ${ }^{6}$ Department of Pediatric Cardiology, Faculty of Medicine, Azad University of Tehran, Tehran, Iran, ${ }^{7}$ Comenius University, Faculty of Natural Science, Department of Geology of Mineral Deposits, Bratislava, Slovakia, ${ }^{8}$ Comenius University, Faculty of Medicine, Institute of Histology and Embryology, Bratislava, Slovakia, and ${ }^{9}$ Comenius University, Faculty of Medicine, Institute of Anatomy, Bratislava, Slovakia

Address for correspondence: C. Biro, MD, Histopatologia Ltd, Antolska 11, SK-851 07 Bratislava, Bratislava. reditary spherocytosis and autoimmune thrombocytopenia and the protective effect of glycoconjugates against overproduction of reactive oxygen species (ROS).

\section{Materials and methods}

Samples

Three samples groups with pathological diagnosis; autoimmune thrombocytopenia (AITP, 5 samples) and hereditary spherocytosis (HS, 5 samples) were taken from human spleen after splenectomy. As reference samples, 5 samples of human spleens from posttraumatic splenectomy with no documented data on spleen related disease were studied.

\section{Light microscopy}

All samples were fixed in $10 \%$ formaldehyde for 24 hours and embedded in paraffin blocks. Thin histological sections (5 $\mu \mathrm{m})$ were prepared using microtome, and mounted on gelatin-coated slides. Sections were stained by haematoxylin and eosine, potassium ferrocyanide (Perls' reaction) for Fe (III) and Alcian blue staining at $\mathrm{pH} 2.5$ for acidic glycoconjugates detection. Samples were examined using Eclipse E50i (Nikon, Japan) light microscope.

\section{Results}

The investigation of Perls' stained slides by light microscope shows iron depositions in both HS and AITP samples, no deposits in the reference group. Positive Perls' reaction with blue stained cytoplasm of macrophages in the splenic cords and in the extracellular space in the samples with HS was observed (Fig. 1). 


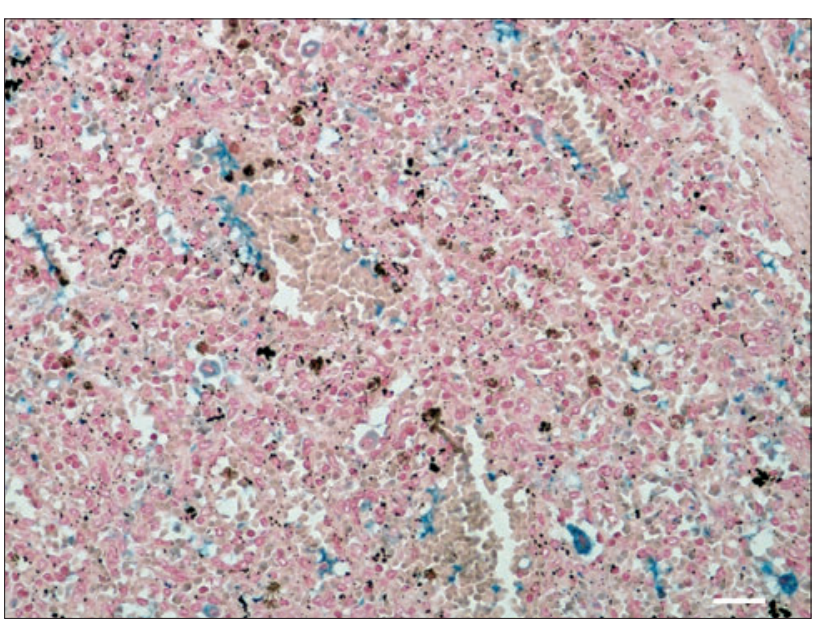

Fig. 1. Light microscopy image of human spleen in hereditary spherocytosis. Blue dyed macrophage cytoplasm in the splenic cords and sporadically in the extracellular space corresponding to $\mathrm{Fe}$ (III) iron (left). The brown to black deposits in the cytoplasm of macrophages corresponding to Fe (II) iron (right), bar, $50 \mu \mathrm{m}$.

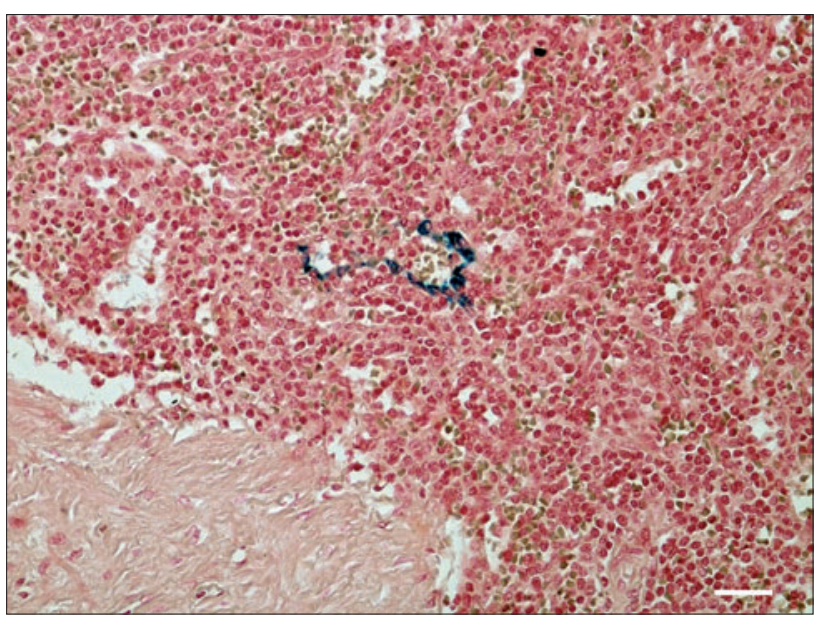

Fig. 2. Light microscopy image of human spleen in autoimmune thrombocytopenia. Sporadically positive Perls' reaction with blue stained cytoplasm of macrophages in the splenic cords and in the extracellular space was observed. Turnbull's reaction imaged the brown to black deposits in the cytoplasm of macrophages. Light microscopy, bar, $50 \mu \mathrm{m}$.

In the AITP group, positive Perls' reaction with blue stained cytoplasm of macrophages in the splenic cords and in the extracellular space was sporadically observed (Fig. 2). A higher positivity of Perls' reaction in the HS group was observed.

Alcian blue staining indicates brown-black spots corresponding to acidic glycoconjugates. A positive AB reaction in cytoplasm of macrophages and in the extracellular space in the samples with HS was observed. The intensity of reaction correlates to the intensity of Pearls' reaction. Physiological erythrocytes corresponded to blue spots (Fig. 3).

Alcian blue staining indicated smaller amount of brown-black material corresponding to acidic glycoconjugates in samples with AITP. Sporadically positive AB reaction was observed in cytoplasm of macrophages and in the extracellular space in the samples

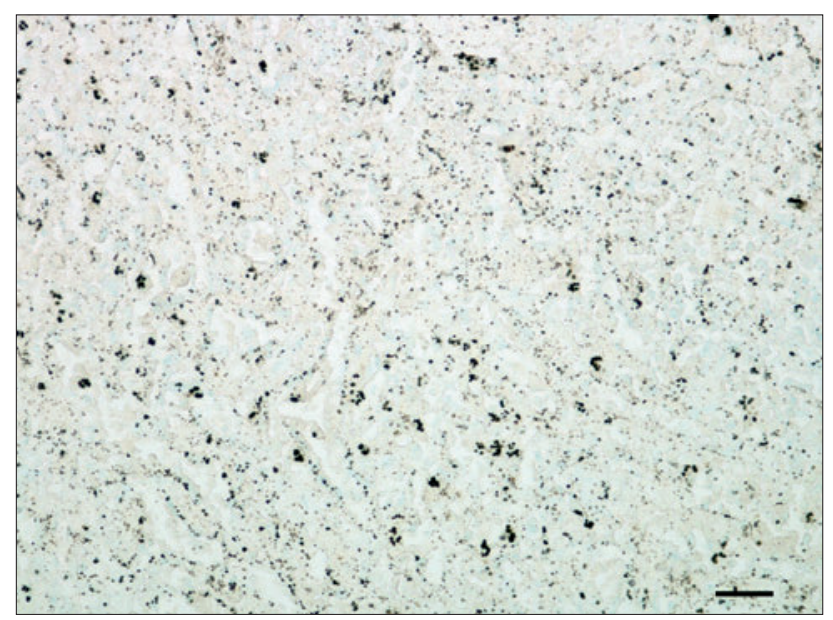

Fig. 3. Light microscopy image of human spleen in hereditary spherocytosis. Erythrocytes correspond to blue spots. Brown-black spots indicate areas corresponding to acidic glycoconjugates. Alcian blue. Bar $=50 \mu \mathrm{m}$.

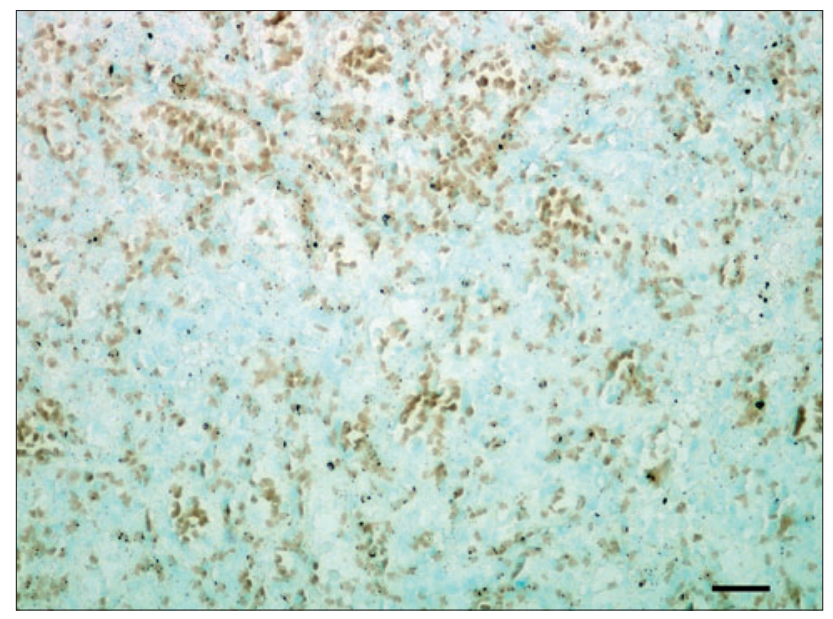

Fig. 4. Light microscopy image of human spleen in autoimmune thrombocytopenia. Blue spots correspond to physiological RBC. Brown spots are erythrocytes after structural change. Alcian blue. Bar $=\mathbf{5 0} \mu \mathrm{m}$.

with AITP. The intensity of reaction correlated to weak intensity of the Pearls' reaction. Physiological erythrocytes corresponded to blue spots, pathological RBC to brown spots. Larger amount of brown-black material was observed in the HS group (Fig. 4).

\section{Discussion}

Iron is an essential element for the many physiological functions of cells, tissues and organs. The largest amount of iron in human body is primarily bound by hemoglobin. It is the iron containing oxygen transport metalloprotein. In the human organism, under physiological circumstances, iron is deposited as ferritin. The ferritin core is composed of Fe (III) (3), coated by organic compounds to avoid ROS generation. Under physiological en- 
vironment, in the spleen old or damaged RBC are destroyed (4) and subsequently engulfed by lysosomes of macrophages (Isom et al, 2009). Under pathological conditions in HS and AITP, higher amounts of RBC are destroyed in human spleen and engulfed by lysosomes of macrophages. Lysosomes are cells' organelles containing various acidic proteins (5). The acidic environment of lysosomes causes permanently ROS formation via Fenton reaction (6). Intralysosomal ROS formation causes lysosomal and cell membrane permeabilization and releasing of iron into intra- and extracellular space (7).

It is well known that ROS play a key role in many pathological processes $(8,9,10)$. The results of ROS overproduction is a damage to biomolecules leading to altered function and disease. Defensive mechanism uses antioxidants as free radical scavengers to neutralise ROS.

Other way of antioxidant defensive mechanism is depositions of organic-inorganic material around iron in order to restrict ROS overproduction. Acidic glycoconjugates demonstrate a high antioxidant ability by strong binding of metal ions (11-16). They create complexes with iron in order to eliminate free iron and thus restrict ROS overproduction $(17,18)$. Iron overload leads to oxidative stress and subsequently to the regulation of antioxidant defenses that may play a major role in resistance to iron-induced oxidative damage (19).

\section{References}

1. Ganesh IM, Subramani D, Halagowder D. Mucin glycoarray in gastric and gallbladder epithelia. J Carcinog 2007; 6: 10.

2. Podracka L, Sasinka M, Boor A, Kovacs L Changes of Physico-chemical characteristics of red blod cells in children with nephrotic syndrome. Internat Urol Nephrol 1996; 28: 593-599.

3. Dobson J Nanoscale biogenic iron oxides and neurodegenerative disease. FEBS Lett 2001; 496: 1-5.

4. Hromec A, Jakubovsky J Spleen, Structure basis of spleen. Ces Physiol 1983; 32: 438-443.

5. Isom HC, McDevitt EI, Moon MS. Elevated hepatic iron: a confounding factor in chronic hepatitis C. Biochim Biophys Acta 2009; 1790: 650662 .

6. Valko M, Morris H, Cronin MT. Metals, toxicity and oxidative stress. Curr Med Chem 2005; 12: 1161-208.

7. Kurz T, Eaton JW, Brunk UT. Redox activity within the lysosomal compartment: Implications for aging and apoptosis. Antioxidants Redox Signal 2010; 13: 511-523.
8. Kravcukova P, Danielisova V, Nemethova M, Burda J, Gottlieb M. Transient forebrain ischemia impact on lymphocyte DNA damage, glutamic acid level, and SOD activity in blood. Cell Mol Neurobiol 2009; 29: 887-894.

9. Soltés L, Mendichi R, Kogan G, Schiller J, Stankovska M, Arnhold J. Degradative action of reactive oxygen species on hyaluronan. Biomacromolecules 2006; 7: 659-668.

10. Bergendi L, Benes L, Duracková Z, Ferencik M. Chemistry, physiology and pathology of free radicals. Life Sci 1999; 65: 1865-1874.

11. Caprari P, Bozzi A, Ferroni L, Strom R, Ropolo M. Oxidative erythrocyte membrane damage in hereditary spherocytosis. Biochem International 1992; 26: 265-274.

12. Presti D, Scott JE. Hyaluronan-mediated protective effect against cell damage caused by enzymatically produced hydroxyl $(\mathrm{OH})$ radicals is dependent on hyaluronan molecular mass. Cell Biochem Funct 1994; 12: 281-288.

13. Albertini R, De Luca G, Passi A, Moratti R, Abuja PM. Chondroitin-4-sulfate protects high-density lipoprotein against copper-dependent oxidation. Arch Biochem Biophys 1999; 365: 143-149.

14. Arai H, Kashiwagi S, Nagasaka Y, Uchida K, Hoshii Y, Nakamura $\mathbf{K}$. Oxidative modification of apoliproprotein $\mathrm{E}$ in human verylow-density lipoprotein and its inhibition by glycosaminoglycans. Arch Biochem Biophys 1999; 367: 1-8.

15. Sela S, Shurtz-Swirski R, Shapiro G, Nasser L, Hamzi M, Shasha SM, Kristal B. Oxidative stress during hemodialysis: Effect of heparin. Kidney Int 2001; 78: S153-S163.

16. Campo GM, Avenoso A, Campo S. Aromatic trap analysis of free radicals production in experimental collagen-induced arthritis in the rat: protective effect of glycosaminoglycans treatment. Free Radic Res 2003; 37: 257-268.

17. Campo GM, D’Ascola A, Avenoso A, Campo S, Ferlazzo AM, Micali C, Zanghi L, Calatroni A. (2004) Glycosaminoglycans reduce oxidative damage induced by Koper $(\mathrm{Cu}+2)$, iron $(\mathrm{Fe}+2)$ and hydrogen peroxide (H2O2) in human fibroblast cultures. Glycoconjugate J 2004; 20: 133-141.

18. Campo GM, Avenoso A, Campo S, D'ascola A, Ferlazzo AM, D Sama, Calatroni A. (2005) Purified glycosaminoglycans limit oxidative injury induced by iron plus ascorbate in skin fibroblast cultures. Toxicol In Vitro 2005; 19: 561-572.

19. Brown KE, Dennery PA, Ridnour LA, Fimmel CJ, Kladney RD, Brunt EM, Spitz DR. Effect of iron overload and dietary fat on indices of oxidative stress and hepatic fibrogenesis in rats. Liver Int 2003; 23 : 232-242.

Received December 6, 2010. Accepted December 18, 2011. 